\section{The Royal College of Ophthalmologists guidelines on serum eye drops for the treatment of severe ocular surface disease: full report}

\section{Introduction \\ Ocular Surface Disease and the Tear Film}

Ocular surface disease (OSD) is a global public health problem with significant impact on quality of life. The ocular surface is a specialised tissue extending from the mucocutaneous junction at the eyelid margin, into the natural gutter (inverse pillar) between the eyelid and eyeball (conjunctival fornix), to the limbus (housing the corneal stem cells) and the cornea (the transparent window in front of the eye). It comprises the surface and glandular epithelia of the cornea, conjunctiva, lacrimal gland, as well as the accessory lacrimal glands, meibomian glands, and their apical (tears) and basal (connective tissue) matrices, and eyelids. ${ }^{1}$ All components of the system are linked functionally by continuity of the epithelia, and their nerve and blood supply together with the endocrine and immune systems. The outer scaffold of the ocular surface is the apical matrix known as the tear film. All regions of the ocular surface epithelia produce constituents of the tear film: the corneal and conjunctival epithelia produce hydrophilic mucins that provide a platform for the aqueous component of the tear film; the lacrimal and accessory lacrimal glands secrete water and protective proteins, immunoglobulins, vitamins, and nutrients vital for ocular surface health, and the meibomian gland provides the complex superficial tear lipid layer that prevents tear evaporation. ${ }^{2}$ These components not only maintain a smooth refractive surface on the cornea to enable sight, the tear film is critical in providing lubrication, physical protection, immunological defence, and nutrition to the ocular surface that is regulated by and closely interacts with the neural, endocrine, vascular, and immune systems.

Failure of one or more of these complex components result in OSD, which in its severest form may lead to blinding complications. These include chronic inflammation, stem cell failure, ulceration, infection, corneal perforation, and scarring. Specifically, conditions that lead to alteration in the production, composition, or distribution of the tear film result in symptoms and signs of damage to the structures of the ocular surface. ${ }^{3}$ The consequence is noticeable irritation, reduction of visual function, severe sight-threatening complications such as infection and corneal perforation, and, importantly, impairment of quality of life similar to that of severe angina, renal dialysis, and disabling hip fracture. ${ }^{4}$ A large number of clinical conditions lead to OSD. These conditions include the following: Sjögren's syndromerelated dry eye, other immune-related dry eye (such as ocular mucous membrane pemphigoid, Stevens-Johnson syndrome, graft-vs-host disease, and ulcerative keratitis), neurotrophic cornea, injury (mechanical, chemical, thermal, and surgery), and stem cell failure.

\section{Current Practice}

Commercially available artificial tears alleviate biomechanical trauma caused by dry eye disease states but lack biological properties such as nutrients that promote ocular surface renewal and immunological defence. This is due to difficulty in synthetically replicating the complex nature of the tear-film architecture and chemical composition.

Lubricants such as those containing

carboxymethylcellulose have improved ocular surface retention and promote epithelial proliferation, whereas sodium hyaluronate preparations exploit the property that it is a ubiquitous naturally occurring extracellular matrix glycosaminoglycan found within the ocular tissues. This has an important role in wound healing, inflammation, and lubrication. Attempts to develop a biological tear substitute that has lubricating and nutrient properties promoting ocular surface renewal and immunological defence have been limited. Isolated reports of single compound topical agents such as Vitamin A, epidermal growth factor, and albumin have sown some in vitro and in vivo efficacy, but clinical response is equivocal and long-term clinical applications have not been developed. Early-phase studies are evaluating the use of amniotic membrane extract or constituent eye drops as a potential alternative.

A variety of the sub-categories of treatments have been recommended by the Dry Eye Workshop II and are listed below. ${ }^{5}$ It should be noted that the evidence for the various options is heterogenous.

- Environmental and dietary advice

- Spectacles and goggles

- Increase humidity

- Reduce exposure to air flow/draft and reduce prolonged visual tasks such as computer work, watching television, and reading

- Omega 3 fish oils

- Omega 7

- Refrain from periocular cosmetics (minimum of 6 weeks trial)

- Lid care

- Warm compresses or proprietary lid warming and expressing devices

- Lid hygiene to reduce lipid biproducts and lipolytic bacteria

- Treatment of bacterial over-colonisaton

- Non-preserved ocular lubricant eye drops and ointments including

- Hypromellose

- Carbomers

- Hydroxypropylguar

- High concentration hyaluronate

- Hyaluronate with xanthangum

- SoyBean with phospholipids

- Ointments 
- Lubricants with osmoprotectants

- Glycerine and L-carnitine and/or erythritol and/or saccharides

- Lubricants and lipids

- Hydroxypropyl guar

- Polar phospholipids

- Mineral oil, soybean oil with phospholipids

- Alternative non-preserved lubricants

- Non-preserved saline $0.9 \%$

- Balanced salt solution

- Tear electrolyte mimetics

- Mucolytics for breakdown of filaments

- Acetylcysteine preserved

- Acetylcysteine non-preserved (UL-HOP)

- Topical anti-inflammatory agents

- Prednisolone non-preserved

- Dexamethasone non-preserved

- Topical ciclosporin (ciclosporin 0.2\% ointment veterinary preparation, ciclosporin $0.1 \%$ (NICE TA269 December 2015)

- Metallomatrix proteinase inhibitors

- Doxycycline-sub anti-microbial dose

- Erythromicin

- Azithromycin

- Punctal occlusion

- Punctal plugs

- Punctal cautery

- Other surgical closure (wounding and suture)

- Secretagogues and stimulants

- Oral pilocarpine (aqueous)

- Oral cevimeline (aqueous-currently not licensed in EU)

- Topical diquafosol tetrasodium (aqueous and mucin - currently not licensed in EU)

- Topical Rebamipide ophthalmic suspension (mucin -currently not licensed in EU)

- Topical testosterone (lipid-currently not licensed in EU)

- Intranasal neurostimulation (FDA approved)

- Contact lenses

- Soft contact lenses

- Rigid gas permeable scleral contact lenses (if Schirmer's I >5 mm)

- Prosthetic replacement of the ocular surface ecosystem (BostonSight PROSE treatment, MA, USA) or similar scleral contact lens

- Blepharospasm

- Botulinum toxin
- 'Topical' biologics

- Recombinant human Nerve Growth Factor $20 \mu \mathrm{g} / \mathrm{ml}$ (marketing authorisation European Medicines Agency July 2017) for neurotrophic keratitis may be useful off license, in neural pain and enhancing goblet cell function

- Lymphocyte function-associated antigen-1 antagonist (Lifitegrast) 5\% (EU license pending)

- Interleukin-1 receptor antagonist (Phase II clinical trials)

A guide to tailoring symptoms and signs of dry eye disease stratified according to disease severity level was originally proposed by the Dry Eye Workshop 2007 (Table 1). ${ }^{6}$ A staged hierarchy of suggested interventions were refined for each level of severity by the Dry Eye Workshop 2017 (Table 2).

\section{Serum Eye Drops}

Physiology. Serum eye drops (SED) are an adjunctive treatment for complex, often immune-mediated, OSD where the production and quality of the tear-film has been compromised leading to debilitating symptoms and severe sight-threatening damage of the surface of the eye; or as supportive therapy for surgical procedures or acute injury (chemical, thermal, and immunological). Serum contains a large number of epitheliotrophic factors that are present in tears and are likely to be responsible for the therapeutic effects observed in patients with OSD over and above conventional commercially available lubricants. 2,3 SED provide the only nutritional tear film substitute available in the United Kingdom that possesses biological properties to help provide an ocular surface environment that promotes epithelial cell renewal and restore homeostasis. This is due to the similarities between the constituents of and the natural (whole) tear film as shown in Table $3 .^{7}$

Since the first reported use of autologous serum eye drop (auto-SED) by Fox in $1984,{ }^{8}$ SED have demonstrated to be effective for the treatment of complex dry eye disease secondary to a wide range of clinical conditions causing OSD (Stevens-Johnson syndrome, Sjögren's syndrome, persistent epithelial defects, graft-vs-host disease, post-LASIK dry eyes, neurotrophic keratopathy, diabetes mellitus, superior limbic keratoconjunctivitis, recurrent corneal erosions, aniridic limbal stem cell deficiency), and supportive therapy for ocular surface reconstruction and stem cell therapy. Demand for the service has been steadily increasing but access to care has been restricted due to a number of multifactorial reasons including licensing status and cost. Finger-prick autologous blood is occasionally used as a cheaper alternative. ${ }^{9}$ The SED treatment is reserved for patients who have severe disease that is refractory to standard interventions, or for those who require supportive therapy for specialised ocular surface surgical procedures, or for use in the acute management of ocular surface injury (chemical, mechanical, thermal, and immunological). 


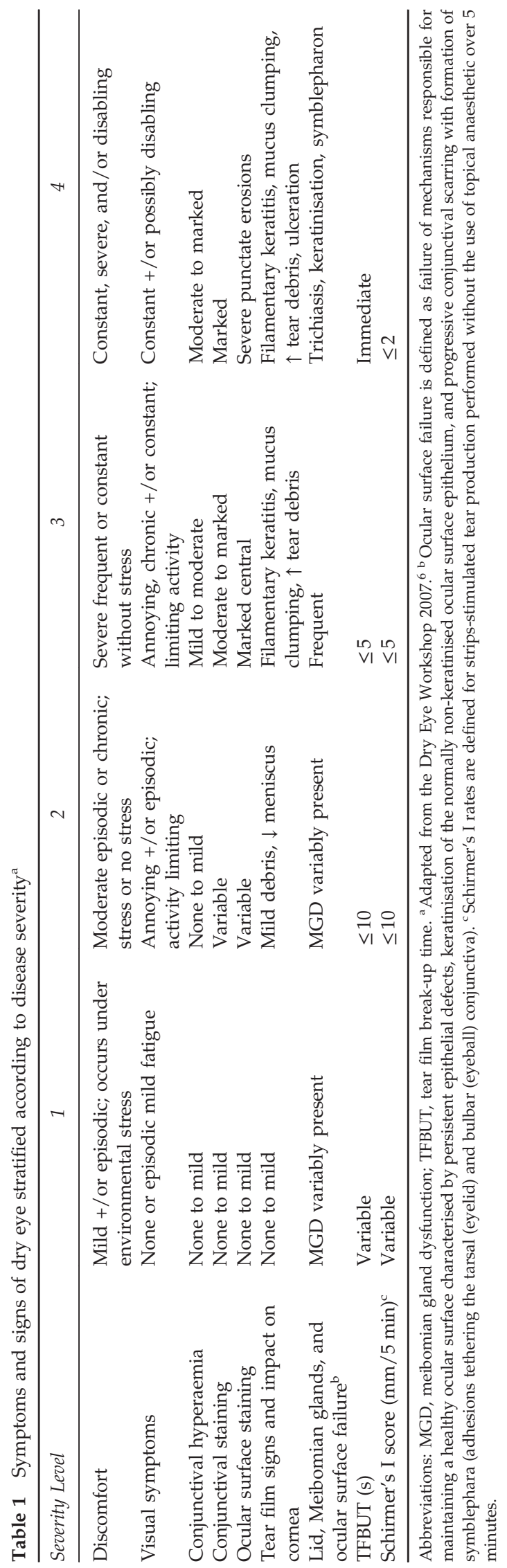

SED service UK. NHS Blood and Transplant Tissue Services (NHSBT) has been providing a SED service since 2003 following the publication from Noble et al. ${ }^{10}$ and prepares SED from the patient's own blood (Auto-SED), and more recently from 2014, from individual (not pooled) male volunteer blood donors (allogeneic serum eye drops (Allo-SED)). SED is an unlicensed medicine that is currently being considered for exclusion from the National Tariff as a High Cost Drug. NHSBT follows strict standard operating procedures. Patients for Auto-SED are required to be of reasonably good health, with no significant cardiovascular or cerebrovascular disease, and free of bacterial infection. Anaemia $(\mathrm{Hb}<11 \mathrm{~g} / \mathrm{dl})$ is a relative contraindication. Allo-SED can be provided for patients who are medically unsuitable to provide an autologous donation. Donations are screened as for hepatitis B and C, HIV I and II, HTLV I and II, and syphilis. One full blood donation produces up to 150 bottles of SED bottles diluted 50\% with saline with a shelf life of 12 months from the date of donation. The majority of the early literature focuses on Auto-SED with recent emergence of interest in Allo-SED. Allo-SED has the advantage of providing treatment if the requirement is immediate or if the patient is unable to donate blood due to their complex medical history (immune-mediated disease, blood cancers, and intensive care patients), poor cardiovascular status, anaemia, and poor venous access. The current patient population eligible for treatment are those with OSD refractory to conventional licensed therapy and those requiring acute management of ocular surface injury and supportive therapy for ocular surface reconstructive procedures.

Outcome measures. Putative data collection tools for baseline and follow-up for both clinical and patientreported outcomes (ocular surface disease index (OSDI)) with a visual analogue scale have been used by NHSBT. Interim data analyses (July 2017) of the OSDI score shows a median reduction in OSDI score of $56.7 \%$, from 57.7 (severe; Q1-Q3 range, 40.6-82.5) pre-commencement of treatment to 25 (moderate; Q1-Q3 range, 12.3-48.6) after 4 months of treatment. Significant improvements are observed in both Allo-SED and Auto-SED groups (Figure 1), and no difference in patient benefit was observed between Allo-SED and Auto-SED.

\footnotetext{
Population

The provision of SED is applicable to any patient with OSD. Children $<16$ years of age are provided with nonCJD risk allogeneic serum imported from Europe. AutoSED is contraindicated in patients who are anaemic, have insufficient venous access, unable to donate the full unit of blood, unable to give consent, and are unconscious or unable to travel to a donor centre. Allo-SED has the advantage of providing treatment if the requirement is immediate or if the patient is unable to donate blood due to their complex medical history (immune-mediated disease, blood cancers, and critical care patients), poor cardiovascular status, anaemia, and poor venous access. A diagnostic breakdown of the population who could potentially benefit from SED is given in Table 4.
} 
Table 2 TFOS Dry eye workshop II (2017) proposed staged management and treatment recommendations $\mathrm{s}^{\mathrm{a}, \mathrm{b}, \mathrm{c}, \mathrm{d}}$

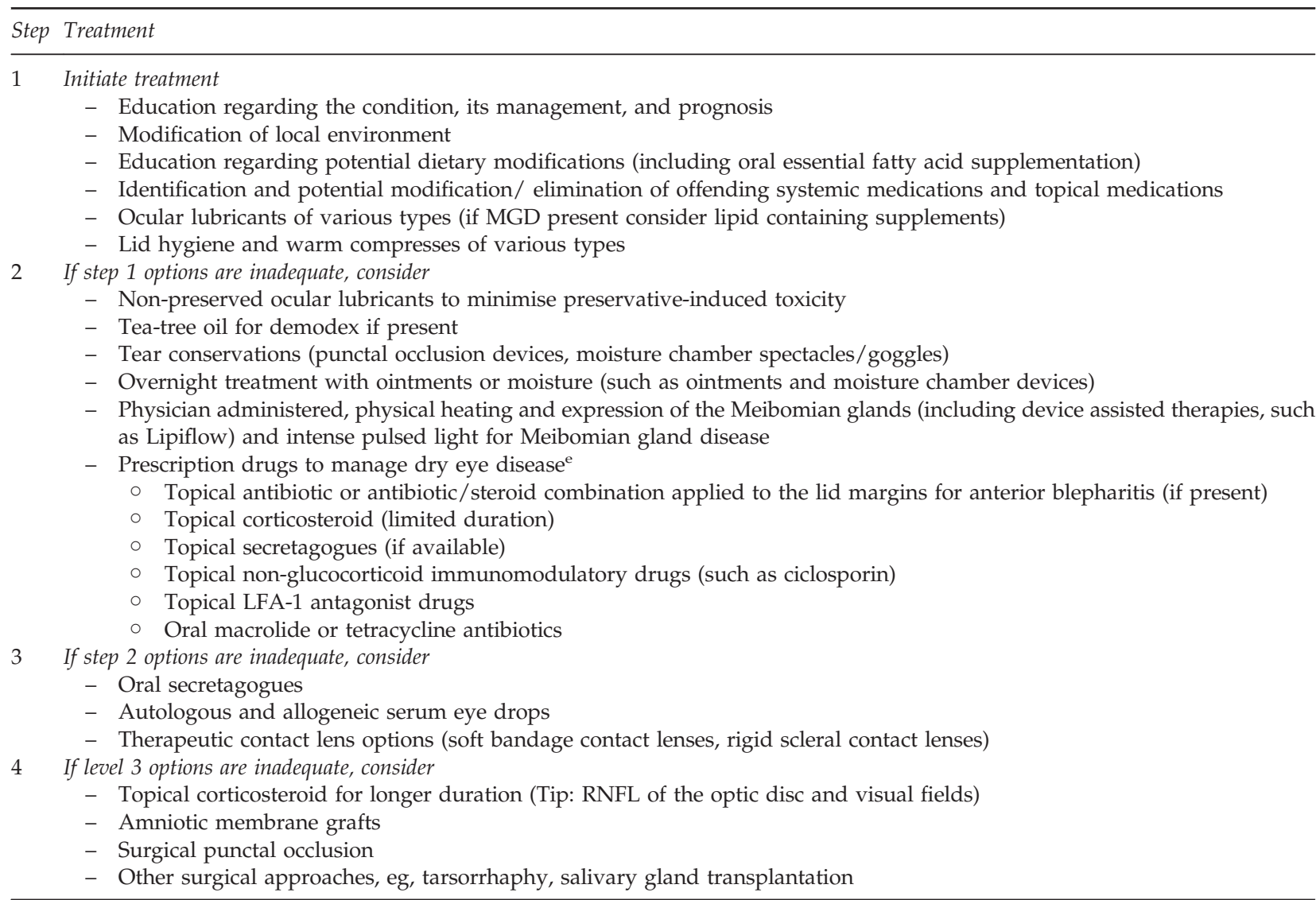

${ }^{a}$ Reproduced and adapted from The Ocular Surface Journal, Dry Eye Workshop II (2017). ${ }^{5}$ botential variations within the disease spectrum are acknowledged to exist between patients and the management options listed above are not intended to be exclusive. The severity and aetiology of the DED state will dictate the range and number of management options selected from one or more steps. ${ }^{c}$ One or more options concurrently within each category can be considered within that step of the dry eye disease state. Options within a category are not ranked according to importance and may be equally valid. ${ }^{\mathrm{d}}$ It should be noted that the evidence available to support the various management options differs and will inevitably be lower for newer management options. Thus, each treatment option should be considered in accordance with the level of evidence available at the time management is instigated. ${ }^{\mathrm{e}}$ The use of prescription drugs needs to be considered in the context of the individual patient presentation, and the relative level of evidence supporting their use for that specific indication, as this group of agents differs widely in mechanism of action.

\section{Scope for change}

SED is a highly specialised, high cost intervention currently classified by the Medicines and Healthcare products Regulatory Agency (MHRA) as an unlicensed medicine. It is reserved for patients with ocular surface or corneal conditions including severe anterior segment inflammation refractory to conventional topical therapy $^{11}$ and other equivalent licensed options. There are no nationally accredited criteria for entering the SED programme and there is an absence of robust systems for recording of outcomes or for implementing withdrawal/stopping strategies. This has led to variation in practice and geographical inequity in access to treatment.

\section{Objectives}

Aims

SED are an unlicensed medicinal product. These

Guidelines aim to provide evidence-based recommendations and good practice points for the safe use of SED for the treatment of severe OSD. They will set out criteria outlining when SED should be considered and provide guidance on how to document outcomes.

Standardising enrolment and clinical documentation will ultimately improve patient safety and care.

\section{The clinical questions covered by the guidelines}

Q1) Are SED more effective at treating patients with OSD than conventional treatment?

Q2) Is there evidence of superiority in the cost and clinical effectiveness of Auto-SED vs Allo-SED at treating patients with OSD?

Q3) What effect does dose size have on the effect of treatment with SED for patients with OSD?

Q4) What effect does concentration of formulation have on the effect of treatment with SED for patients with OSD?

Q5) What effect does duration of treatment have on the effect of treatment with SED for patients with OSD? 
Table 3 Similarities of key constituents in whole tears and serum (reproduced from Rauz and Saw) ${ }^{7}$

\begin{tabular}{lcc}
\hline Parameter & Whole tears & Serum \\
\hline $\mathrm{pH}$ & 7.4 & 7.4 \\
Osmolality & 298 & 296 \\
EGF $(\mathrm{ng} / \mathrm{ml})$ & $0.2-3.0$ & 0.5 \\
TGF- $\beta(\mathrm{ng} / \mathrm{ml})$ & $2-10$ & $6-33$ \\
NGF $(\mathrm{pg} / \mathrm{ml})$ & 468.3 & 54.0 \\
IGF $(\mathrm{ng} / \mathrm{ml})$ & 0.31 & 105 \\
PDGF $(\mathrm{ng} / \mathrm{ml})$ & 1.33 & 15.4 \\
Albumin $(\mathrm{mg} / \mathrm{ml})$ & 0.023 & 53 \\
Substance P $(\mathrm{pg} / \mathrm{ml})$ & 157 & 70.9 \\
Vitamin A $(\mathrm{mg} / \mathrm{ml})$ & 0.02 & 46 \\
Lysozyme $(\mathrm{mg} / \mathrm{ml})$ & 1.4 & 6 \\
Surface $\mathrm{IgA}(\mu \mathrm{g} / \mathrm{ml})$ & 1190 & 2 \\
Fibronectin $(\mu \mathrm{g} / \mathrm{ml})$ & 21 & 205 \\
Lactoferrin $(\mathrm{ng} / \mathrm{ml})$ & 1650 & 266 \\
\hline
\end{tabular}

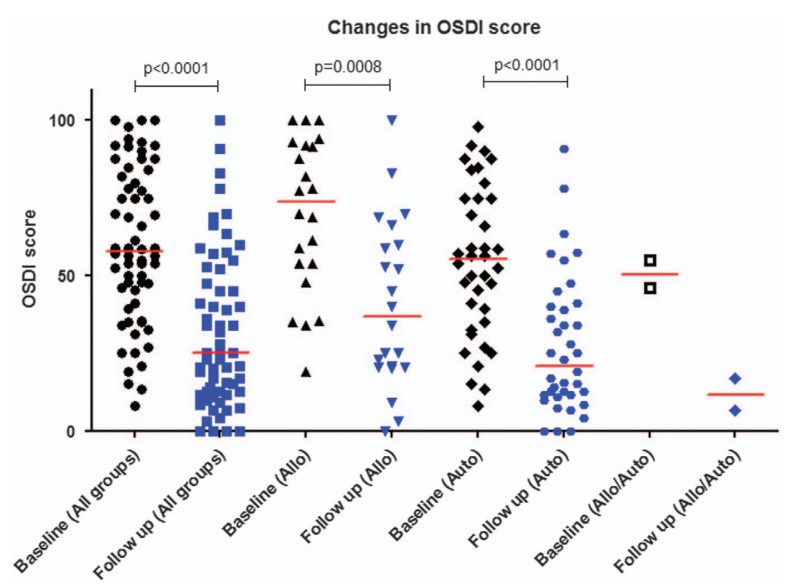

Figure 1 Impact of SED therapy on the OSDI score. (All patients, $n=62$; Allo, $n=22$; Auto, $n=38$; Allo/Auto (patients initiated on Allo-SED for speed then switched to Auto-SED), 2; Pvalues derived from Wilcoxon matched-pairs signed-rank test).

Q6) What effect does frequency of treatment have on the effect of treatment with SED for patients with OSD?

Q7) Which clinical outcome measures best record the treatment effect for monitoring OSD?

Q8) Which patient reported outcome measures best record the treatment effect for monitoring impact on patient debility?

\section{Description of the key stakeholders and end users}

Target audience:. Ophthalmologists (Consultants and Specialty and Associate Specialist doctors) caring for adults and children with OSD in secondary and tertiary care

\section{Other beneficiaries:}

- Multi-professional teams who have patients with ocular surface manifestations of systemic diseases including Haematologists, Rheumatologists, Neurologists,
Table 4 Examples of the patient populations who may benefit from SED therapy ${ }^{a}$

\begin{tabular}{ll}
\hline Main category & Examples \\
\hline Sjögren's Syndrome & $\begin{array}{l}\text { Primary Sjögren's Syndrome } \\
\text { Secondary Sjögren's Syndrome }\end{array}$
\end{tabular}

Other immune-related ocular surface disease

Ocular mucous membrane pemphigoid Stevens-Johnson syndrome/ toxic epidermal necrolysis Graft-vs-host disease Other immune-related ocular surface disease

Neurotrophic disease

Diabetic cornea

Herpetic aetiology

Other neuropathic disease including secondary to nonocular, extra-ocular and neurosurgical causes

$\begin{array}{ll}\text { Injury } & \text { Ocular surface toxicity } \\ & \text { Chemical } \\ & \text { Thermal } \\ & \text { Mechanical } \\ & \text { Radiation } \\ & \text { Surgical } \\ & \text { Other Injury } \\ & \text { Ocular surface reconstruction } \\ & \text { Corneal transplantation } \\ & \text { Other supportive, eg, critical } \\ & \text { care unit/high dependency/ } \\ \text { burns unit } & \\ & \text { Aniridia } \\ \text { Ectodermal dysplasia } & \text { Epidermolysis bullosa } \\ \text { Inherited ocular surface disease } & \text { Other inherited ocular disease }\end{array}$

${ }^{a}$ This is not a comprehensive list. Examples are given for guidance only.

Dermatologists, General Physicians, and General Practitioners who will review patients with OSD.

- Healthcare professionals and practitioners such as those working in Intensive Care Medicine, Specialist Nurses, Optometrists, and Orthoptists.

- The guideline should also be of relevance to Specialist Trainees.

- Commissioners and providers of services for adults and children with OSD.

- Adults and children with OSD and their families and carers.

\section{Stakeholders:}

- Royal College of Ophthalmologists

- The Bowman Club

- NHSBT

- Ocular Tissue Advisory Group 
- Serum Eye Drops Patient Support Group

- British Society of Blood and Marrow Transplant

- British Society of Rheumatology

- British Sjögren's Syndrome Association

\section{Methods}

Methodology

This guideline has been developed in accordance with the Guideline Development Manual of The Royal College of Ophthalmologists (found at RCOphth.ac.uk) following the pre-specified stages to ensure that the recommendations are aligned with the strength of evidence available from the review of the literature.

\section{Search strategy}

Key questions for the guideline were developed using the Patient, Intervention, Comparison and Outcome (PICO) framework to provide a structured basis for identifying the evidence. A systematic review of the literature was undertaken using the explicit search strategies devised in collaboration with the Cochrane Eyes and Vision Group. Databases searched include Medline, Embase, and the Cochrane Library for literature published between 1992 and 2017. Further searches were undertaken on various websites including the US National Guidelines Clearinghouse. All PICO search strategies used are shown in Supplementary Appendix 1.

The evidence base for this guideline was identified and synthesised in accordance with the accepted methodology with each of the selected papers was evaluated by two members of the group using standard checklists before conclusions were considered as acceptable evidence. The literature search focused on the best available evidence to address the key review questions by including the following types of evidence:

- Published guidelines

- Systematic reviews

- Randomised controlled trials

- Cohort and case control studies

\section{- Case series}

Papers not published in the English language, conference abstracts, and letters were excluded.

\section{Levels of Evidence and Grades of Recommendations} Evidence was graded by the Guideline Development Group according to its strength using the Scottish Intercollegiate Guidelines Network framework (SIGN 50 -Table 5). The strength of each recommendation took into account the quality of the evidence.

Using the evidence identified, the Guideline Development Group determined the guideline recommendations. The strength of each recommendation was based upon the quality of the evidence and the potential for patient benefit.

This guideline makes a strong recommendation where:

- The evidence is of high quality

- Estimates of the effect of an intervention are precise (ie, there is a high degree of certainty that effects will be achieved in practice)

- There are few downsides of therapy

- There is a high degree of acceptance among patients

And a conditional recommendation is made where:

- There are weaknesses in the evidence base

- There is a degree of doubt about the size of the effect that can be expected in practice

- There is a need to balance the upsides and downsides of therapy

- There are likely to be varying degrees of acceptance among patients

The strength of the recommendation has been graded by the Guideline Development Group using the methodology from the SIGN 50. The grade of recommendation relates to the strength of the evidence on which the recommendation is based (Table 6). It does not reflect the clinical importance of the recommendation.

Table 5 Scottish Intercollegiate Guidelines Network framework (SIGN 50)

\begin{tabular}{|c|c|}
\hline $\begin{array}{l}\text { Type of } \\
\text { evidence }\end{array}$ & Description \\
\hline $1++$ & High-quality meta-analyses, systematic reviews of RCTs, or RCTs with a very low risk of bias \\
\hline $1+$ & Well-conducted meta-analyses, systematic reviews of RCTs, or RCTs with a low risk of bias \\
\hline $1-$ & Meta-analyses, systematic reviews of RCTs, or RCTs with a high risk of bias* \\
\hline \multirow[t]{2}{*}{$2++$} & High-quality systematic reviews of case-control or cohort studies \\
\hline & $\begin{array}{l}\text { High-quality case-control or cohort studies with a very low risk of confounding, bias or chance, and a high probability } \\
\text { that the relationship is causal }\end{array}$ \\
\hline $2+$ & $\begin{array}{l}\text { Well-conducted case-control or cohort studies with a low risk of confounding, bias or chance and a moderate probability } \\
\text { that the relationship is causal }\end{array}$ \\
\hline 2- & $\begin{array}{l}\text { Case-control or cohort studies with a high risk of confounding, bias, or chance and a significant risk that the relationship } \\
\text { is not causal }\end{array}$ \\
\hline 3 & Non-analytic studies (eg, case reports, case series) \\
\hline 4 & Expert opinion, formal consensus \\
\hline
\end{tabular}


Table 6 Grade of recommendation

\begin{tabular}{|c|c|}
\hline Grade & Explanation \\
\hline A & $\begin{array}{l}\text { At least one meta-analysis, systematic review, or RCT rated as } 1++ \text {, and directly applicable to the target population, or } \\
\text { A body of evidence consisting principally of studies rated as } 1+\text {, directly applicable to the target population, and demonstrating } \\
\text { overall consistency of results } \\
\text { A body of evidence including studies rated as } 2++ \text {, directly applicable to the target population, and demonstrating overall } \\
\text { consistency of results, or extrapolated evidence from studies rated as } 1++ \text { or } 1+\end{array}$ \\
\hline B & $\begin{array}{l}\text { A body of evidence including studies rated as } 2+\text {, directly applicable to the target population and demonstrating overall } \\
\text { consistency of results, or extrapolated evidence from studies rated as } 2++\end{array}$ \\
\hline $\mathrm{C}$ & $\begin{array}{l}\text { Evidence level } 3 \text { or } 4 \text {; or } \\
\text { Extrapolated evidence from studies rated as } 2+\end{array}$ \\
\hline GPP & Good practice points based upon consensual expert opinion where the evidence base does not support A-C grading \\
\hline MHRA & Medicines and Healthcare products Regulatory Agency Guidance Note 14 \\
\hline $\mathrm{R}$ & Further research is required in this area \\
\hline
\end{tabular}

\section{Results \\ Q1) Are SED more effective at treating patients with OSD, than conventional treatment?}

Scope. There is wide consensus among specialists in OSD that SED have a role in the treatment of disorders such as severe immune-mediated dry eye disease, persistent and recurrent corneal epithelial defect, neurotrophic keratopathy, and patients who require supportive therapy post ocular surface reconstruction. The treatment is reserved for those who are unresponsive or partially responsive to available licensed conventional therapeutic options considered to be appropriate for the patient. The evidence underpinning the benefit of treating patients with OSD and whether SEDs are more effective than those on conventional treatment was reviewed.

Evidence. A Cochrane review on the use of SED in adults with dry eye was published in $2017^{12}$ and summarised the results of five RCTs (Celebi et al, ${ }^{13}$ Kojima et al, ${ }^{14}$ NodaTsuruya et al, ${ }^{15}$ Tananuvat et al, ${ }^{16}$ and Urzua et al ${ }^{17}$ ). They concluded that SED 20\% may provide some benefit in improving patient-reported symptoms in the short term ( 2 weeks), but that there appears to be no evidence of improvement over a longer period. Of note, there was unclear evidence to suggest improvement for objective measures of the OSD. The authors recommended that further large, high-quality RCTs using standardised questionnaires, objective clinical tests and objective biomarkers are warranted to assess the benefit of SED in the longer term. (Evidence 1++, Positive/Equivocal).

Another systematic review ${ }^{18}$ evaluated the use of blood derived topical therapy (including SED) in OSD, concluding that the use of SED in dry eye disease improved OSDI scores, fluorescein staining score, and TBUT, as well as reducing concurrent use of topical lubricants. SED also appeared to be effective in the treatment of PED, but study numbers were small. (Evidence 1++, Positive/Equivocal).

Although there is paucity of strong supporting evidence, several reviews have reported a trend for superiority of SED in alleviating some of the clinical signs and symptoms in Sjögren's ${ }^{19}$ and non-Sjögren's dry eye disease, limbal epithelial stem cell deficiency, Graft-vs-Host-Disease, persistent epithelial defects, recurrent corneal erosions, post-refractive surgery, ${ }^{20}$ and Stevens-Johnsons syndrome/toxic epidermal necrolysis. ${ }^{21}$ A double masked RCT in 40 eyes $^{13}$ demonstrated improved tear film stability and patient comfort with SED, supported by three other case series in 123 eyes of 63 dry eye patients, ${ }^{22} 56$ eyes of 28 patients, ${ }^{23}$ and in 17 patients with graft-versus-host disease. ${ }^{24}$ In another RCT, Improved recovery time from corneal abrasions induced during vitrectomy surgery has also been shown. ${ }^{25}$

A publication by a panel of North American experts on the management of dry eye in Sjögren's syndrome ${ }^{26}$ corroborated the recommendation for the use of serum drops by the 2007 International Management and Therapy Subcommittee of the International Dry Eye Workshop I and II. 5,6 These publications acknowledged the limited evidence available, but recommended use of SED for severe dry eye unresponsive to conventional measures.

Table 7 shows a summary of all the studies included in the evidence review.

In the United Kingdom, the NHSBT is conducting an evaluation of new patients who are enrolled onto the SED programme. These data, capture baseline and follow up findings at 4 months. Data being collected include patient perceptions of their disease using a validated tool (OSDI) and clinical findings using a corneal function score (Oxford Ocular Surface Grading System) together with tear film break-up time and Schirmer's test without anaesthetic, both performed using DEWS 2007 methodology. The results are pending but these should provide useful information on the effectiveness of SED among patients with OSD and will form the largest published case series using Auto-SED and Allo-SED.

Recommendation: SED will benefit:

1) Patients who have refractory or partially responsive acute or chronic severe OSD where licensed interventions have been considered (Recommendation grade A).

2) Patients with other ocular surface conditions such as recurrent corneal erosions, persistent epithelial defects and limbal epithelial stem cell failure may benefit if licensed interventions have been unsuccessful (Recommendation grade B). 


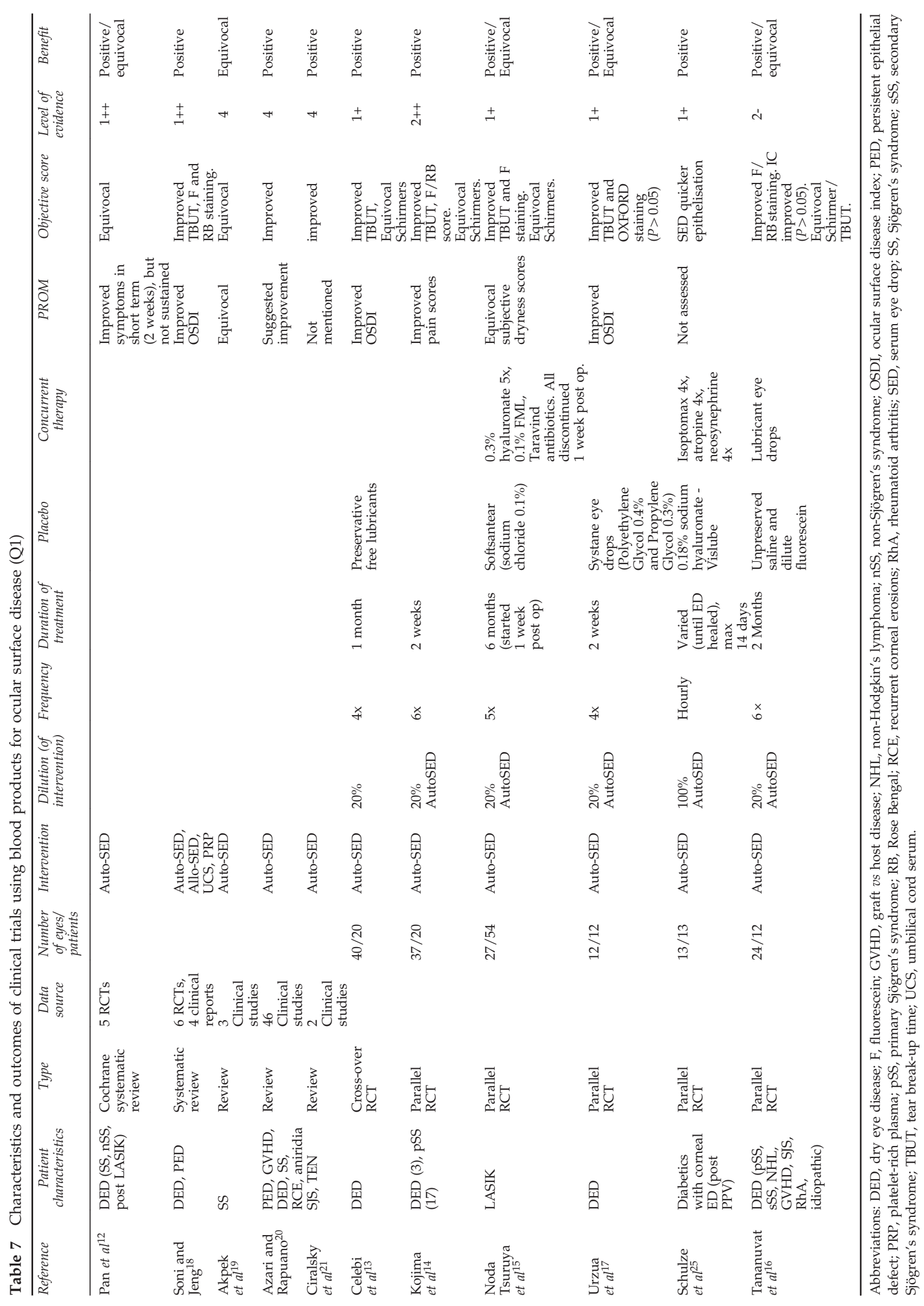


3) Patients who require supportive therapy such as for patients undergoing ocular surface reconstruction (Recommendation grade B)

\section{Q2) Is there evidence of superiority in the cost and clinical effectiveness of Auto-SED versus Allo-SED at treating patients with OSD?}

Scope. Patients with inflammatory OSD frequently have systemic manifestations, which preclude the ability to donate blood for the production of autologous serum, require Allo-SED. This may be due to general health issues such as patients who have poor venous access and patients who are unable to attend an apheresis/donor centre. Such patients include those patients with poor mobility, eg, multiple sclerosis, rheumatic disease and those in critical care situations.

The evidence interrogating efficacy, superiority and cost-benefit of Allo-SED versus Auto-SED in these situations was evaluated.

Evidence. There are no studies that have performed a direct comparison of effectiveness of Auto-SED versus Allo-SED in the treatment of OSD. Lekahnont et al. ${ }^{27}$ performed a prospective study of 181 eyes, where 178 were received Auto-SED and 3 Allo-SED. However, given the large discrepancy in group sizes, this was not felt to be a valid comparison study.

There are also no published studies comparing the direct and indirect cost effectiveness of Auto-SED vs AlloSED, or studies that determine the impact of the economic benefit or burden of the treatment. In the United Kingdom, the NHSBT provides Auto-SED and Allo-SED at a cost of approximately $£ 1,100$ for 3-5 months' supply (including delivery to the patient's home address with same day courier).

\section{Recommendation:}

1) Auto-SED should be considered for patients who are fit to donate one unit of blood, are able to travel to a blood donor centre, or the patient prefers SED to be made from their own blood (Recommendation grade GPP)

2) Allo-SED should be considered in patients who are unable to donate one unit of blood such as those who are in poor general health, unable to attend a blood donor centre, less than age 16 years, or there is a clinical requirement for urgent treatment (Recommendation grade GPP)

3) Clinical trials comparing the clinical efficacy and cost effectiveness of Auto-SED vs Allo-SED are required (Recommendation grade R)

\section{Q3) What effect does dose size have on the effect of} treatment with SED for patients with OSD?

Scope. SED provide a physiological tear substitute for patients with OSD with nutritional properties in addition to reduction of biomechanical trauma or friction provided by commercially available substitutes. Published comparisons of serum, plasma, and tear components highlight similarities between these biofluids but do not provide ranges to gauge the bio-variability of each constituent within Auto-SED vs the natural tear film. The composition of the Auto-SEDs made from donations from patients with diabetes, immune-mediated diseases, those on cytotoxic drugs, or with sepsis will have potentially harmful serum constituents that could lead to severe ocular surface toxicity. Patients with underlying immunemediated disease may have circulating antibodies, growth factors, and pro-inflammatory cytokines within their serum that theoretically may exacerbate disease if administered topically to the surface of the eye. These patients may benefit from Allo-SED rather than AutoSED. Patients in this grouping include those with graft-vshost disease, acute toxic epidermal necrolysis, or mucous membrane pemphigoid.

The evidence regarding the biological variability of the composition of Auto-/Allo-SED and the dose of each blood constituent that is optimal for a therapeutic effect, $v s$ the dose that could lead to ocular surface toxicity, was considered to be important. The literature in this area was reviewed.

Evidence. There are no published clinical trials that specifically compare the effects of individual constituents in SED for the treatment of OSD. This includes serum levels of putative toxins, although studies evaluating the pathophysiology of various OSDs highlight systemic activation of the adaptive immune response and subsequent expression of proinflammatory mediators at the ocular surface. ${ }^{3}$

\section{Recommendation:}

1) Allo-SED should be considered as an option in patients with uncontrolled diabetes, refractory immunemediated diseases, those on cytotoxic agents or where their bi-products are known to damage proliferating cells (eg, cyclophosphamide) and patients with sepsis (Recommendation grade GPP)

2) Detailed serum constituent analyses of sequential donations from patient and healthy donors is required to interrogate bio-variability of each donation and the impact this could have on ocular surface health (Recommendation grade $\mathrm{R}$ )

3) Further work on the development of protocols that reduce variability of biological constituents is required, eg, pooling of serum samples from multiple donors with measured ranges of main constituents. (Recommendation grade $\mathrm{R}$ )

Q4) What effect does concentration of formulation have on the effect of treatment with SED for patients with OSD?

Scope. Published data shows the similarities between tear and serum constituents. Manufacturing process 
(clotting time, centrifuging, temperature, and so on) varies from country to country and there appears to be no internationally agreed standard operating procedures for the production of SED. ${ }^{28}$ This includes whether it is clinically more effective to treat patients with SED manufactured without dilution and delivered as 100\% serum, or if diluted, what is the optimal diluent or carrier and concentration to achieve the desired clinical effect.

Evidence. Published studies have used varying concentrations of SED: most commonly $20 \%$, followed by $50 \%$ and $100 \%$ (Table 7 ). SEDs are usually diluted with sodium chloride $0.9 \%$ to achieve the desired final product concentration.

There is only one published study (Cho et al), ${ }^{29}$ which compared the efficacy of SED with different diluents in patients with dry eyes (Sjögren's syndrome and nonSjögren's syndrome) and persistent epithelial defects. In this study, SED were administered as: 100\% serum, 50\% serum with $0.9 \% \mathrm{NaCl}, 50 \%$ serum with sodium hyaluronate $0.3 \%$, or $50 \%$ serum with ceftazidime $5 \%$. The authors concluded that $100 \%$ SED helped to improve subjective symptoms and objective findings in both Sjögren's and non-Sjögren's dry eye, and increases healing speed in eyes with persistent epithelial defects. However, in the non-Sjögren's dry eye group, 50\% SED (diluted with $0.9 \% \mathrm{NaCl}$ ) showed similar improvements as $100 \%$ SED. The authors also reported that SED diluted in $0.9 \% \mathrm{NaCl}$ showed the best effects, and despite their expectations, there was no synergistic effect of hyaluronic acid when used at a diluent for SED. SED diluted with ceftazidime was found to be least effective due to the antibiotics own epithelial toxicity. The results of this study are summarised in Table 8.

\section{Recommendation:}

The use of Auto-SED and Allo-SED as a 50\% dilution in $0.9 \%$ sodium chloride is recommended (as provided by NHSBT, the only accredited SED production facility in the UK) (Recommendation grade: GPP)

\section{What effect does duration (Q5) and frequency (Q6) of treatment have on the effect of treatment with SED for patients with OSD?}

Scope. Clinical guidance given to patients on how frequently they should administer SED and for what duration varies from patient to patient, and their underlying clinical condition. Some patients commence treatment for a short duration (1-2 donations providing 4-6 months treatment) on a 2 hourly basis to determine whether a high pulse of topical treatment may induce remission whilst others are on life-long treatment. The evidence for optimal duration and frequency, and indication for when to stop treatment was considered to be important.

Evidence. No studies have examined optimal frequency and/or duration of SED therapy for a specific clinical 
indication. There are no studies evaluating when it might be safe to stop SED therapy.

There are considerable variations in treatment frequency in published studies (Tables 7 and 8: $4 \times /$ day to hourly usage). There is no clear evidence to suggest that more frequent instillation results in improved subjective symptoms and objective clinical findings. Similarly, the optimal duration of treatment with SED is unclear due to heterogeneity in the published studies. The duration of treatment in studies ranges from 2 weeks to 6 months-this however often coincides with the study duration, and it is unclear how many patients continue on SED after conclusion of the study.

\section{Recommendation:}

1) Frequency and duration of treatment depends upon individual circumstances. The doctor responsible for patient care should consider withdrawal and stopping strategies in all patients commenced on SED treatment before committing patients to indefinite treatment. Such strategies may include (i) withdrawal of treatment after one year of therapy in patients with OSD, to define induction of remission before reinstating indefinite treatment if symptoms relapse, or (ii) in patients with persistent corneal epithelial defects, withdrawal of treatment after surface of the eye has healed and restoring treatment if the surface shows signs of breakdown. (Recommendation grade GPP)

2) Further research is required on the optimal formulation and diluent. This includes considering whether a $100 \%$ formulation is as effective as one that is diluted. A search for vehicles or carriers that improve the retention time and patient satisfaction is recommended. (Recommendation grade $\mathrm{R}$ )

3) Further work is required on the frequency and duration of SEDs treatment used for each clinical indication. Clinical trials should specifically consider when it might be safe to implement treatment withdrawal in patients who have achieved measured success or remission according to pre-set defined criteria. (Recommendation grade R)

Which clinical $(Q 7)$ outcome or patient reported (Q8) outcome measures best record the treatment effect for monitoring OSD and the impact on patient debility? Scope. Consistent recording of clinical and patient reported outcomes enables a unified approach to objective assessment of treatment response to novel or highly specialised interventions such as SEDs. The generation of cohort registries and datasets (as recommended by the Quality, Innovation, Productivity, and Prevention programme) facilitates the quantification of efficacy in a clinical setting, serious adverse events, and ultimately the impact of SED on the health economic burden.

Nevertheless, it is recognised that patient perceptions of disease influencing severity scoring outweigh observed clinical signs in some patients with ocular neuropathic pain. The presence and validity of published clinical and patient reported outcome instruments for use in monitoring the clinical effect of SED for standardisation of outcome reporting and patient benefits, was determined.

Evidence. There is a heterogeneity in outcome reporting in the monitoring of the effects of SED. There are no studies that have specifically validated objective scores for clinical examination findings (ocular surface staining score, Schirmer's test, tear film break-up time), laboratory investigations (impression cytology, surface expression markers, blood, or urine tests), nor patient reported outcome measures (visual analogue scales, OSDI, 5-item Dry Eye Questionnaire (DEQ-5), and visual function questionnaires) for recording the treatment effect of SED. Given the absence of specific information,

recommendations are extrapolated from generic tools used for patients with OSD and as recommended by DEWS II, diagnostic methodology algorithm. ${ }^{30,31}$

\section{Recommendation:}

1) Instruments for assessment of the impact of treatment on health-related quality of life and objective grading of patient perceptions of disease using utility instruments specific for OSD, should be considered for use regularly in the clinical setting. These include the OSDI or the shorter DEQ-5. (Recommendation grade GPP)

2) Consistent recording of clinical outcome measures and scoring of disease should be considered. This includes visual acuity, meniscus height, presence of filaments, tear film break-up time, ocular surface staining score, eg, Ocular Staining Score, epithelial defect measurements (if present), and Schirmer's test without anaesthetic (Recommendation grade GPP)

3) It is advised that patients treated with Auto-SED and Allo-SED should be enrolled into a national programme. Frequency and duration of treatment together with serious adverse events should be recorded using a standard reporting procedure. A minimum follow-up of 6 months and then annually should be considered (Recommendation grade GPP)

4) Development and validation of SED-specific patient reported outcome tools and minimal clinical datasets for efficient outcome reporting is required. (Recommendation grade $\mathrm{R}$ )

\section{Good Practice Points and Recommendations}

SED are an unlicensed medicinal product. These guidelines recommend that SED are beneficial for patients with acute and chronic severe OSD including patients with severe dry eye, persistent and recurrent corneal epithelial defects, neurotrophic keratopathy, and for patients requiring supportive therapy for surgery (see 


\section{Quick Guideline Reference for the Use of Serum Eye Drops in Severe Ocular Surface Disease}
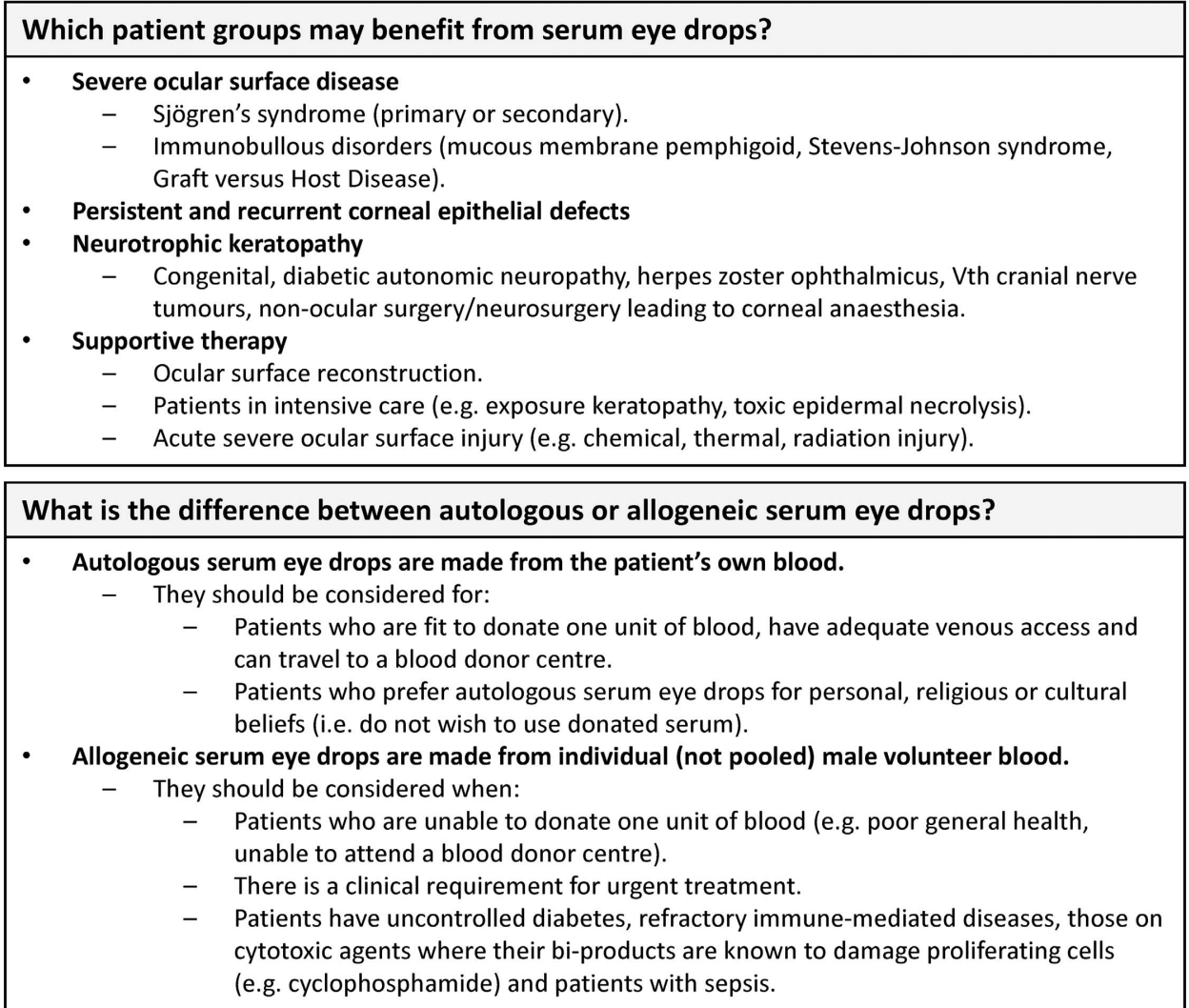

How should serum eye drops be prescribed?

- Serum Eye Drops are an MHRA unlicensed medicine ('special'), and should only be prescribed after licensed treatments have been considered or have been unsuccessful.

- Patient's disease severity should be defined with subjective and objective parameters, and be monitored throughout treatment to determine treatment response. It is recommended that monitoring occurs both locally and in a centralised registry.

- Autologous and Allogeneic Serum Eye Drops as a $\mathbf{5 0 \%}$ dilution in $\mathbf{0 . 9 \%}$ Sodium chloride are recommended (as provided by the NHSBT, the only accredited production facility in the UK).

- Frequency and duration of treatment depends upon individual circumstances.

- Withdrawal and stopping strategies should be considered in all patients commenced on serum eye drops treatment before committing patients to indefinite treatment. For example:

- In ocular surface disease: Withdrawal of treatment after one year of therapy to define induction of remission before reinstating indefinite treatment if symptoms relapse.

- In persistent corneal epithelial defects: Withdrawal of treatment after surface of the eye has healed and restoring treatment if the surface shows signs of breakdown.

Figure 2 Quick reference guide for the use of autologous and allogeneic serum eye drops in severe ocular surface disease.

Quick Guideline Reference (Figure 2)). Severity should be defined with subjective and objective parameters and licensed treatments should be carefully considered before SED are prescribed. Monitoring of treatment effect with both patient and clinical reported outcomes is essential with specific consideration given for implementing treatment withdrawal and stopping strategies. Good practice includes clinical audit to document efficacy, safety, adverse reactions, and collection of data through a centralised patient registry to monitor longer term outcomes. Registry development and integration of direct and indirect costs to define effectiveness of treatment is recommended. Further research is required to determine bio-substance variability in serum donations, potential toxicity of autologous drops in some patients, identification of biomarkers for monitoring effectiveness, and determining optimal frequency, dosing and duration of SED treatment for each indication. 


\section{Clinical Indications for SED Treatment}

- MHRA regulatory note: SED are an unlicensed medicine. In accordance to the MHRA Guidance Note 14 (2014), supply of unlicensed medicinal products ('specials'), anyone supplying an unlicensed medicinal product, where an equivalent licensed medicinal product is available must be satisfied as to the existence of a special need for the unlicensed medicinal product. MHRA expects that documentary evidence of this special need should be obtained by manufacturers, importers or distributors and that this evidence should be made available on request of the Licensing Authority.

- Severe OSD: most common in Sjögren's syndrome (both primary and secondary to connective tissue diseases typically rheumatoid arthritis and systemic lupus erythematosis), immunobullous disorders usually mucous membrane pemphigoid, Stevens-Johnson syndrome, Graft-vs-Host Disease.

- Persistent and recurrent corneal epithelial defects: caused by dry eye disease, as well as other inflammatory ocular surface conditions, commonly severe allergic eye diseased, following corneal infections, limbal epithelial stem cell failure, neurotrophic keratitis.

- Neurotrophic keratopathy: this may be congenital, secondary to diabetic autonomic neuropathy, herpes zoster ophthalmicus, Vth cranial nerve tumours, non-ocular surgery/neurosurgery leading to corneal anaesthesia.

- Supportive therapy: for ocular surface reconstruction, patients in an intensive care setting with acute exposure keratopathy or toxic epidermal necrolysis, and those presenting with severe ocular surface injury such as chemical, thermal, or radiation injury.

\section{Patients Not Suitable for SED}

- MHRA regulatory note: SED should not be supplied where an equivalent licensed medicinal product can meet the special needs of the patient. Responsibility for deciding whether an individual patient has 'special needs', which a licensed product cannot meet should be a matter for the doctor responsible for the patient's care. Examples of 'special needs' include an intolerance or allergy to a particular ingredient

- Patients who have mild to moderate disease are not suitable for SED

\section{Eligibility Criteria}

All patients should meet clinically defined severity criteria according to the primary disease process and the doctor responsible for the management of the patent should have considered other available reversible causes / contributory factors and available licensed treatment options. The criteria described in the NHSE Specialised Service Circular SSC1728 March 2017 should be followed and are summarised as below:

Guidance for defining disease severity:

- Severe, persistent ocular surface symptoms for $>1$ year

- Patient severity score

- Visual Analogue Score (0-10): $>8$

- OSDI (Max 100): > 33

- Tear film break-up time: $<3 \mathrm{~s}$

- Staining domains:

- Van Bjisterveld score $($ Max 9) $=8$ to 9

- Ocular Surface Staining Score (Max 12) =9 to 12

- Oxford Staining Score (Max 15) =11 to 15

- Persistent epithelial defect unresponsive to standard treatment

Therapeutic options prior to commencing SED Treatment for patients with OSD should begin by implementing conservative self-help options and supplementary tears with non-preserved artificial substitutes, tear modification with acetylcysteine, where possible tear stimulation with pilocarpine, disease modification with anti-inflammatories, and surface modification strategies. If there is absence of significant relief for the patient as measured by clinical and patientreported outcomes, SED may be considered as a therapeutic option. A combination of the following treatment approaches should be considered. Please note the management options are not exhaustive.

- Conservative and environmental modification:

- Local and general environmental modifications (eg, moist chamber goggles, workplace modification including humidifiers)

- Nutritional supplements such as Omega 3 and Omega 7

- All comorbidities should be considered and optimised including lid margin disease, lid malposition, trichiasis, and blepharospasm

- Lubricants and tear substitution:

- Basic lubricant preparations such as hypromellose, carbomer gels, and ointments

- Regular, frequent non-preserved ocular lubricant including hydroxypropylguar, hyaluronates, and hyaluronate combinations (carboxymethylcellulose, polysaccharide, disaccharide or xanthan gum, soybean with phospholipids)

- Tear modification:

- Acetylcysteine

- Osmoprotectants

- Liposomal sprays 
- Tear stimulation:

- Use of secretagogues in incremental doses may be beneficial in selected cases, eg, oral pilocarpine

- Disease modification:

- Topical anti-inflammatories such as non-preserved topical glucocorticoids, topical calcineurin inhibitors (NICE TA369 December 2015)

- Systemic disease modifiers such as metallomatrix proteinase inhibitors, eg, sub-anti-microbial dose of antibiotics including low dose of tetracyclines and macrolides

- Punctal occlusion:

- Punctal plugs and permanent cautery or occlusion (lower lid and then upper lid)

\section{- Surface modification:}

- Where possible therapeutic contact lenses (eg, rigid gas permeable, soft hydrogel) provide a protective barrier to the ocular surface

- Rigid gas permeable scleral contact lenses are vaulted away from the ocular surface supported by the anterior sclera, enable a pre-corneal therapeutic reservoir to be created

Specific considerations. For other clinical conditions such as recurrent corneal erosions, persistent epithelial defects, limbal epithelial stem cell failure, and ocular surface reconstruction, consider use of therapeutic contact lenses, corneal epithelial debridement, amniotic membrane graft to the cornea (including PROKERA (Biotissue, FL, USA), OmniLenz (NuVision Biotherapies Ltd, Nottingham, UK)), phototherapeutic laser, and medical or surgical tarsorrhaphy as appropriate.

\section{Autologous vs Allogeneic Treatment}

1) Auto-SED should be considered for patients who are fit to donate one unit of blood, are able to travel to a blood donor centre, or the patient prefers auto-SED for personal, religious, or cultural beliefs, and do not wish to use donated serum.

2) Allo-SED should be considered in patients who are unable to donate one unit of blood such as those who are in poor general health, unable to attend a blood donor centre, less than age 16 years, or there is a clinical requirement for urgent treatment.

3) Allo-SED should be considered as an option in patients with uncontrolled diabetes, refractory immunemediated diseases, those on cytotoxic agents where their bi-products are known to damage proliferating cells (eg, cyclophosphamide), and patients with sepsis.

4) Frequency and duration of treatment depends upon individual circumstances. The doctor responsible for patient care should consider withdrawal and stopping strategies in all patients commenced on SED treatment before committing patients to indefinite treatment. Such strategies may include (i) withdrawal of treatment after 1 year of therapy in patients with OSD to define induction of remission before reinstating indefinite treatment if symptoms relapse, or (ii) in patients with persistent corneal epithelial defects, withdrawal of treatment after surface of the eye has healed and restoring treatment if the surface shows signs of breakdown. These are examples and this list should not be considered as exhaustive.

\section{Monitoring}

Treatment effect should be monitored with both patient and clinical reported outcome instruments both locally and in a centralised registry. This is essential for determining long-term clinical and cost effectiveness of treatment. A web-based quality dashboard is considered best practice. Such tools detail frequency and duration of treatment together with record of serious adverse events using standard reporting procedures. Given the absence of evidence, a follow-up of 6 months and annual review should be considered.

Health-related quality of life burden increases with the severity of disease, although disproportionate symptoms to signs (ocular neuropathic pain) is recognised. Objective grading of patient perceptions of disease using patientreported outcome utility instruments specific for OSD is recommended, eg, the OSDI tool (Supplementary Appendix 2). This is a 12-item questionnaire sub-divided into 3 domains: visual function (6); ocular symptoms (3); environmental triggers (3) where $0=$ no disability and $100=$ complete disability, or the shorter DEQ-5.

It is recommended that objective baseline clinical outcome tools are used. These tools should attempt to capture patient demographics, as well as scoring of clinical signs to document response to treatment. Such data should include ethnicity and residential post code, centre details, confidentiality statement, date of treatment, clinical indication, type of SED treatment (autologous and allogeneic), clinical outcome measures and scores (visual acuity, meniscus height, presence of filaments, tear film osmolarity, tear film break-up time, ocular surface staining score, epithelial defect measurements (if present), and Schirmer's test together with guides to standardise clinical methodology to record outcomes. (Supplementary Appendix 3).

The follow-up outcome tool should be implemented after about 4-6 months of treatment. It should capture additional information such as whether the patient is still on treatment, has been transferred to another hospital, whether the treatment has been discontinued and whether there have been adverse local reactions or events (Supplementary Appendix 4). There should be a record of whether other treatments (such as lubricants) were continued, reduced or withdrawn as they were no longer required. Ideally, longer term outcome data (1 year) to determine duration of treatment, or what 
proportion of patients are on indefinite duration treatment is required.

Recording of outcomes is a changing field. The example documents (Supplementary Appendicies 2, 3 and 4) are currently implemented by NHSBT. These will undergo controlled updates and if scales come into greater use and are practically validated, then they will be incorporated in the revised versions.

\section{Further research}

The Guidelines development group recognises the need for further research. Several areas where further clinical trials or laboratory analyses have been identified are listed below. This list is not exhaustive.

1) Clinical trials comparing the clinical efficacy and cost effectiveness of autologous SED vs allogeneic SED are required.

2) Detailed serum constituent analyses of sequential donations from patient and healthy donors is required to interrogate bio-variability of each donation and the impact this could have on ocular surface health.

3) Further work on the development of protocols that reduce variability of biological constituents is required, eg, pooling of serum samples from multiple donors with measured ranges of main constituents.

4) Further research is required on the optimal formulation and diluent. This includes considering whether a 100\% formulation is as effective as one that is diluted. A search for vehicles or carriers that improve the retention time and patient satisfaction is recommended.

5) Further work is required on the frequency and duration of SED treatment in each clinical indication. Clinical trials should specifically consider when it might be safe to implement treatment withdrawal in patients who have achieved measured success or remission according to pre-set defined criteria.

6) Development and validation of SED-specific patient reported outcome tools and minimal clinical datasets for efficient outcome reporting is required.

\section{Conflict of interest}

Dr A Chandrasekar and Dr R Lomas represented the NHS Blood and Transplant Tissue Services (accredited suppliers of the serum eye drops service in the UK) in the Guidelines Development Group. The remaining authors declare no conflict of interest.

\section{Author contributions}

The Multidisciplinary team involved in producing these guidelines was chaired by Miss Saaeha Rauz (Consultant Ophthalmologist specialising in OSD), who proposed and led the development of the guidelines. Miss Su-Yin Koay was involved in the grading of the evidence and providing summary tables. Mr Barny Foot represented the Royal College of Ophthalmologists Quality Team supported the guidance development. Professor Stephen Kaye and Professor Francisco Figueiredo provided Ocular Surface Specialist clinical input and Mr Michael Burdon provided general ophthalmology input and assured the guidance was produced in accordance to the Royal College of Ophthalmologists Guidance Development Manual. Dr Akila Chandrasekar and Dr Richard Lomas represented NHS Blood and Transplant Tissue Services and provided source data from the SED service. Mrs Elizabeth Dancey contributed as a Patient and Carer representation and heads the Serum Eye Drops Patient Focus and Support Group.

\section{Acknowledgements}

The Royal College of Ophthalmologists would like to thank the Cochrane Eyes and Vision Group and in particular Iris Gordon for her assistance in searching the evidence base. The development of this guideline was funded by The Royal College of Ophthalmologists. This guidance was peer-reviewed through a formal consultation process where members of the Royal College of Ophthalmologists, Bowman Club, Ocular Tissue Advisory Group, and British Society of Blood and Marrow Transplant were invited to provide comments. The draft guidance was available for wider consultation through publication on the Royal College of Ophthalmologists website and final version was ratified through the Scientific Committee at the Royal College of Ophthalmologists.

\section{References}

1 Gipson IK. The ocular surface: the challenge to enable and protect vision: the Friedenwald Lecture. Invest Ophthalmol Vis Sci 2007; 48(10): 4391-4398.

2 Willcox MDP, Argüeso P, Georgiev GA et al. TFOS DEWS II Tear Film Report. Ocul Surf 2017; 15(3): 366-403.

3 Bron AJ, de Paiva CS, Chauhan SK et al. TFOS DEWS II pathophysiology report. Ocul Surf 2017; 15(3): 438-510.

4 Buchholz P, Steeds CS, Stern LS et al. Utility assessment to measure the impact of dry eye disease. Ocul Surf 2006; 4(3): 155-161.

5 Jones L, Downie LE, Korb D et al. TFOS DEWS II management and therapy report. Ocul Surf 2017; 15(3): 575-628.

6 Management and Therapy of Dry Eye Disease: Report of the Management and Therapy Subcommittee of the International Dry Eye WorkShop (2007). Ocul Surf 2007; 5(2): 163-178.

7 Rauz S, Saw VP. Serum eye drops, amniotic membrane and limbal epithelial stem cells-tools in the treatment of ocular surface disease. Cell Tissue Bank 2010; 11(1): 13-27.

8 Fox RI, Chan R, Michelsen JB et al. Beneficial effect of artificial tears made with autologous serum in patients with keratoconjunctivitis sicca. Arthritis Rheum 1984; 27(4): 459-461.

9 Than J, Balal S, Wawrzynski J et al. Fingerprick autologous blood: a novel treatment for dry eye syndrome. Eye 2017; e-pub ahead of print 16 June 2017; doi:10.1038/eye.2017.118.

10 Noble BA, Loh RSK, MacClennan S et al. Comparison of autologous serum eye drops with conventional therapy in a 
randomised controlled crossover trial for ocular surface disease. Br J Ophthalmol 2004; 88: 647-652.

11 NHS England Standard Contract Service Specification for Specialised Ophthalmology (Adult, Ref: D12/S/a,Oct/2013).

12 Pan Q, Angelina A, Marrone $\mathrm{M}$ et al. Autologous serum eye drops for dry eye. Cochrane Database Syst Rev 2017; 2: CD009327.

13 Celebi AR, Ulusoy C, Mirza GE. The efficacy of autologous serum eye drops for severe dry eye syndrome: a randomized double-blind crossover study. Graefes Arch Clin Exp Ophthalmol 2014; 252: 619-626.

14 Kojima T, Ishida R, Dogru M et al. The effect of autologous serum eyedrops in the treatment of severe dry eye disease: a prospective randomized case-control study. Am J Ophthalmol 2005; 139(2): 242-246.

15 Noda-Tsuruya T, Asano-Kato N, Toda I et al. Autologous serum eye drops for dry eye after LASIK. J Refract Surg (Thorofare, NJ: 1995) 2006; 22(1): 61-66.

16 Tananuvat N, Daniell M, Sullivan LJ et al. Controlled study of the use of autologous serum in dry eye patients. Cornea 2001; 20(8): 802-806.

17 Urzua CA, Vasquez DH, Huidobro A et al. Randomized double-blind clinical trial of autologous serum versus artificial tears in dry eye syndrome. Curr Eye Res 2012; 37(8): 684-688.

18 Soni NG, Jeng BH. Blood-derived topical therapy for ocular surface diseases. Br J Ophthalmol 2016; 100(1): 22-27.

19 Akpek EK, Lindsley KB, Adyanthaya RS et al. Treatment of Sjogren's Syndrome-associated dry eye: an evidence-based review. Ophthalmology 2011; 118(7): 1242-1252.

20 Azari AA, Rapuano CJ. Autologous serum eye drops for the treatment of ocular surface disease. Eye Contact Lens 2015; 41(3): 133-140.

21 Ciralsky JB, Sippel KC, Gregory DG. Current ophthalmologic treatment strategies for acute and chronic Stevens-Johnson syndrome and toxic epidermal necrolysis. Curr Opin Ophthalmol 2013; 24(4): 321-328.

22 Hussain M, Shtein RM, Sugar A et al. Long-term use of autologous serum $50 \%$ eye drops for the treatment of dry eye disease. Cornea 2014; 33(12): 1245-1251.

23 Liu Y, Hirayama M, Cui X et al. Effectiveness of autologous serum eye drops combined with punctal plugs for the treatment of Sjogren syndrome-related dry eye. Cornea 2015; 34(10): 1214-1220.

24 Tahmaz V, Gehlsen U, Sauerbier L et al. Treatment of severe chronic ocular graft-versus-host disease using 100\% autologous serum eye drops from a sealed manufacturing system: a retrospective cohort study. Br J Ophthalmol 2016.

25 Schulze SD, Sekundo W, Kroll P. Autologous serum for the treatment of corneal epithelial abrasions in diabetic patients undergoing vitrectomy. Am J Ophthalmol 2006; 142(2): 207-211. http: / / onlinelibrary.wiley.com/o/cochrane/ clcentral/articles/928/CN-00566928/frame.html.

26 Foulks GN, Forstot SL, Donshik PC et al. Clinical guidelines for management of dry eye associated with Sjogren disease. Ocul Surf 2015; 13(2): 118-132.

27 Lekhanont K, Jongkhajornpong P, Choubtum L et al. Topical $100 \%$ serum eye drops for treating corneal epithelial defect after ocular surgery.[Erratum appears in Biomed Res Int. 2014;2014:595973; doi: 10.1155/2014/595973]. BioMed Res Int 2013; 2013: 521315.

28 Geerling G, MacClennan S, Hartwig D, Autologous Serum Eye. Drops for ocular surface disorders. Br J Ophthalmol 2004; 88: 1467-1474.

29 Cho YK, Huang W, Kim GY et al. Comparison of autologous serum eye drops with different diluents. Curr Eye Res 2013; 38(1): 9-17.

30 Methodologies to Diagnose and Monitor Dry Eye Disease: Report of the Diagnostic Methodology Subcommittee of the International Dry Eye WorkShop (2007). Ocul Surf 2007; 5(2): 108-152.

31 Wolffsohn JS, Arita R, Chalmers R et al. TFOS DEWS II diagnostic methodology report. Ocul Surf 2017; 15(3): 539-574.

S Rauz ${ }^{1}$, S-Y Koay ${ }^{2,3}$, B Foot ${ }^{2}$, SB Kaye ${ }^{4}$, F Figueiredo ${ }^{5}$, MA Burdon ${ }^{2,6}$, E Dancey ${ }^{7}$, A Chandrasekar ${ }^{8}$ and R Lomas ${ }^{8}$

${ }^{1}$ Academic Unit of Ophthalmology, Institute of Inflammation and Ageing, Birmingham and Midland Eye Centre and University of Birmingham, Birmingham, UK

${ }^{2}$ Royal College of Ophthalmologists, London, UK

${ }^{3}$ Moorfields Eye Hospital, London, UK

${ }^{4}$ Royal Liverpool University Hospital, Liverpool, UK

${ }^{5}$ Newcastle Royal Victoria Infirmary, Newcastle, UK

${ }^{6}$ Queen Elizabeth Hospital Birmingham, Birmingham, UK

${ }^{7}$ Serum Eye Drops Patient Focus and Support Group, Birmingham, UK

${ }^{8} \mathrm{NHS}$ Blood and Transplant Tissue Services, Liverpool, UK E-mail: s.rauz@bham.ac.uk

Eye advance online publication, 17 November 2017; doi:10.1038/eye.2017.209

Supplementary Information accompanies this paper on Eye website (http://www.nature.com/eye) 